\title{
SPECTROSCOPIC RADIAL VELOCITIES: PHOTOSPHERIC LINESHIFTS CALIBRATED BY HIPPARCOS
}

\author{
D. GULLBERG, D. DRAVINS \\ Lund Observatory, Box 43, SE-22100 Lund, Sweden
}

Wavelengths of stellar spectral lines depend not only on the star's motion. Until recently, accurate studies of shifts not caused by radial motion were feasible only for the Sun. Solar lineshifts are interpreted as gravitational redshift $(636 \mathrm{~m} / \mathrm{s})$ and convective blueshifts $(\sim 400 \mathrm{~m} / \mathrm{s}$; caused by velocity-brightness correlations). In other stars, such effects may be greater (Dravins \& Nordlund 1990). Accurate astrometric radial velocities are now available from Hipparcos (Dravins et al. 1997a; $1997 \mathrm{~b}$ ), permitting studies of such shifts also in some other stars.

For such stars in the open clusters of Hyades, Ursa Major and Coma Berenices, a spectroscopic program is in progress, analyzing wavelength shifts in groups of lines with different strengths, excitation potentials, etc., using the ELODIE high-precision radial-velocity instrument (Baranne et al. 1996) at Haute-Provence Observatory.

\section{References}

Baranne, A., et al. (1996) A\&AS 119,373

Dravins, D., Nordlund, $\AA$. (1990) A\&A 228, 203

Dravins, D., Lindegren, L., Madsen, S., Holmberg, J. (1997a), HIPPARCOS Venice '97, ESA SP-402, p. 733

Dravins, D., Lindegren, L., Madsen, S., Holmberg, J. (1997b) Highlights of Astronomy 11 (this volume)

Further project description: http://www.astro.lu.se/ dainis/

\section{ASTROMETRIC RADIAL VELOCITIES FROM HIPPARCOS}

\author{
D. DRAVINS ${ }^{1}$, L. LINDEGREN ${ }^{1}$, S. MADSEN ${ }^{1,2}$, J. HOLMBERG ${ }^{1}$ \\ ${ }^{1}$ Lund Observatory, Box 43, SE-22100 Lund, Sweden \\ ${ }^{2}$ Copenhagen University Observatory, J. Maries Vej 30, 2100 Copenhagen, Denmark
}

Space astrometry now permits accurate determinations of stellar radial motion, without using spectroscopy. Although the feasibility of deducing astrometric radial velocities from geometric projection effects was realized already by Schlesinger (1917), only with Hipparcos has it become practical. Such a program has now been carried out for the moving clusters of Ursa Major, Hyades, and Coma Berenices. Realized inaccuracies reach about $300 \mathrm{~m} / \mathrm{s}$ (Dravins et al. 1997).

Discrepancies between astrometric and spectroscopic radial velocities reveal effects (other than stellar motion) that affect wavelength positions of spectral lines. Such are caused by stellar surface convection, and by gravitational redshifts. A parallel program (Gullberg \& Dravins 1997) is analyzing high-precision spectroscopic radial velocities for different spectral lines in these stars, using the ELODIE radial-velocity instrument at Haute-Provence.

\section{References}

Dravins, D., Lindegren, L., Madsen, S., Holmberg, J. (1997) ESA SP-402, p. 733

Gullberg, D., Dravins, D. (1997) Highlights of Astronomy 11 (this volume)

Schlesinger, F. (1917) $A J$ 30, 137

Further project description: http://www.astro.lu.se/ dainis/ 